\title{
Replantations des cocoteraies : mythe ou réalité ?
}

\section{Replanting coconut plantations: myth or reality?}

Oléagineux, Corps Gras, Lipides. Volume 7, Numéro 2, 182-8, Mars - Avril 2000, Dossier : Afrique, plantation et développement

Auteur(s) : Vincent RIBIER, André ROUZIERE

Résumé : Le constat du faible dynamisme des plantations de cocotier et de la part déclinante du coprah dans la production et les échanges mondiaux pourrait laisser craindre que les cocoteraies sont condamnées à terme. Cela n'est pas si sûr, car le cocotier dispose d'un certain nombre d'atouts, notamment en conditions villageoises, et se prête à des utilisations variées qui dépassent le strict cadre du coprah : la plante dite aux mille usages est d'abord une plante vivrière. L'article passe en revue les conditions sous lesquelles de nouvelles plantations sont envisageables et présente quelques solutions alternatives à la replantation, comme la réhabilitation ou la diversification dans le cadre d'associations de culture sous cocotier adulte.

Summary: The article examines the future of coconut plantations worldwide, and more specifically in Africa. It does so by retracing planting dynamics over a long period (1960-1999), attempting to explain the main trends, describing the main conditions under which coconut planting or rehabilitation are feasible, and proposing a methodological approach designed to identify appropriate action plans for the problems encountered. Whilst the sluggishness of coconut planting and the declining share of copra in world trade are mentioned, the article also draws attention to the different functions fulfilled by coconut plantings in medium and small-scale production structures. In this context, targeted replanting or rehabilitation programmes can have a highly positive impact on the well-being of the populations involved, especially when they form part of a production diversification initiative, with integration of derived or alternative activities.

Keywords: coconut plantations, rehabilitation and replanting programmes, coconut planting dynamics, methodological approach.

\section{ARTICLE}

\section{Coup d'arrêt aux plantations et replantations de cocoteraies dans les années 90}

Les statistiques de superficie sous cocotier sont assez imprécises en Afrique, ce qu'illustre bien le cas de la Côte d'Ivoire. Pour la FAO, la surface cumulée de la cocoteraie ivoirienne est passée de 4 000 à 53000 hectares entre 1961 et 1994, avant de redescendre vers 30000 ha à partir de 1995, et ce sans raison apparente, alors qu'Oil World continue [1], quant à lui, de créditer ce pays d'une superficie de 50000 hectares sous cocotier. Les données présentées dans le tableau 1 sont donc à prendre avec précaution. 
Les superficies totales cultivées en cocotier ont nettement augmenté au cours des 40 dernières années : elles sont passées de 5,23 millions d'hectares en 1961 à près de 10,5 millions d'hectares en 1999. Cette progression s'est toutefois ralentie au fil des décennies, indiquant que les nouvelles plantations sont devenues de plus en plus rares sur la période.

Cette tendance mondiale est également observable en Afrique : la superficie des cocoteraies africaines est passée de 430000 à 664000 ha entre 1961 et 1999, mais la majeure partie de cette extension s'est réalisée au cours des années 60 et 70 . II n'y a pratiquement plus de nouvelles plantations en Afrique depuis 1990. Les dynamiques de plantation ont été très variables selon les pays, et il est possible de distinguer trois cas de figure :

- les pays pour lesquels la cocoteraie, déjà importante en 1960, a connu une forte expansion avant 1990 : c'est notamment le cas de la Tanzanie et du Ghana ; la croissance de la cocoteraie tanzanienne est telle qu'elle représente actuellement près de la moitié des plantations africaines ;

- les pays où le cocotier, marginal avant 1960, a bénéficié d'un important développement au cours des 30 années suivantes (Madagascar et, surtout, Côte d'Ivoire) ;

- les pays n'ayant connu aucune dynamique de plantation entre 1960 et 2000 : il s'agit notamment du Mozambique, du Nigeria et de la plupart des autres pays producteurs africains.

Les plus fortes progressions de superficies sous cocotier ont été réalisées dans le cadre de plans cocotier mis en œuvre entre 1965 et 1990 par certains États qui avaient entrepris une politique de développement volontariste. Des plans cocotier ont ainsi été mis en œuvre en Côte d'Ivoire, à Madagascar, en Tanzanie et au Ghana. Les autres pays africains ne sont que peu intervenus dans le développement de leur filière cocotier nationale.

La dynamique d'accroissement des superficies de cocoteraies est quasiment stoppée depuis 1990. Les nouvelles plantations sont très rares : les différents plans cocotier qui avaient permis de nets accroissements de superficie plantée dans les années 60,70, voire 80, n'ont plus été poursuivis dans les années 90. Cela entraîne un vieillissement du verger africain et, en conséquence, une chute de la productivité. Dans ce contexte de retrait des interventions publiques volontaristes, les petits planteurs n'ont pas pris le relais et l'on n'observe que de très rares replantations villageoises. Ce phénomène préoccupant de non-renouvellement affecte la plupart des cultures pérennes, notamment dans le cas des plantations paysannes : les petits planteurs, qui ont généralement bénéficié d'un crédit (société de plantation, aide de l'État) au moment de la plantation, ont rarement les moyens de supporter le coût d'une replantation. Ainsi, en 1994, près du tiers de la palmeraie africaine cultivée était âgée de plus de 25 ans, c'est-à-dire qu'elle se situait au-delà du cycle normal d'exploitation économique. La situation est identique dans le cas de l'hévéa, l'essentiel des replantations concernant les ensembles agro-industriels.

\section{Quelques éléments d'explication}

Le fort ralentissement des dynamiques de plantation/replantation en cocoteraies traduit le déclin du coprah vis-à-vis des autres oléagineux. La part du coprah dans l'ensemble des huiles végétales est en effet passée de 7,6 \% en 1961 à seulement 2,7 \% en 1999 (tableau 2). 
Ce processus de marginalisation est dû tout à la fois à la substitution progressive de l'huile de coprah par celle de palme pour les usages alimentaires en zone tropicale [2] et à la montée en puissance de l'huile de palmiste qui vient concurrencer l'huile de coprah à l'intérieur des huiles lauriques pour les usages industriels et cosmétiques [3]. L'huile de palmiste, très minoritaire au sein des lauriques au début des années 60 (production de 490000 tonnes contre 1860000 tonnes pour I'huile de coprah), fait maintenant presque jeu égal avec l'huile de coprah. Les exportations d'huiles lauriques suivent une évolution similaire : dynamisme du palmiste et morosité de l'huile de coco. Les exportations d'huile de coco stagnent depuis 1950. Une stabilisation récente des exportations d'huile de palmiste est toutefois observée, du fait du développement des capacités de l'oléochimie en Malaisie qui absorbe ainsi des quantités croissantes d'huile de palmiste pour son marché intérieur au détriment de ses exportations.

Différents facteurs concourent au déclin du coprah.

\section{Baisse de la productivité}

La productivité agronomique du cocotier n'a connu aucune amélioration au cours des quarante dernières années, alors que celle des autres graines oléagineuses a augmenté dans des proportions parfois importantes. Les itinéraires techniques en cocoteraies ont peu évolué, très peu d'innovations techniques importantes ayant été transférées de la recherche à la production (diffusion très limitée des hybrides haut producteurs, par exemple), si bien que la production de noix par hectare a plutôt eu tendance à baisser sur la période du fait du vieillissement des plantations. Par ailleurs, la production du coprah n'est que très faiblement mécanisable, la plupart des opérations telles que celles du débourrage et du décoquage restant exclusivement manuelles. En conséquence, la production d'huile par hectare de cocoteraie a baissé depuis 1960, alors qu'elle a été multipliée par cinq dans le cas du palmier (tableau 3).

\section{Structure des exploitations et de la transformation}

La production de coprah est assez dispersée et provient davantage de petites exploitations qui cultivent conjointement au moins un autre produit que de grandes plantations industrielles, tandis que les plantations de palmiers sont le fait de grandes exploitations spécialisées dans cette culture. Les conditions de transformation sont également à l'avantage du palmier : la production d'huile alimentaire à base de coco est souvent réalisée par une petite industrie locale, souvent artisanale, alors que le traitement de l'huile de palme est concentré dans quelques grosses usines, ce qui facilite son contrôle et sa distribution.

\section{Forte variabilité des rendements}

L'huile de coprah est largement pénalisée par la plus grande variabilité de sa production. Le coprah est susceptible de connaître des écarts de rendement importants d'une année sur l'autre, bien supérieurs à ceux observés dans le cas des autres graines oléagineuses. Cette situation, à l'origine de fortes et fréquentes tensions sur les approvisionnements, a des conséquences néfastes sur les anticipations des agents. Les fluctuations en volume sont en effet perçues comme un risque par les utilisateurs qui préfèrent se tourner vers des produits concurrents dont l'approvisionnement est plus régulier. 


\section{Diverses fonctions de la cocoteraie}

L'analyse précédente, axée sur une valorisation des cocoteraies par le seul coprah, ne promet qu'un avenir assez sombre pour la culture du cocotier. À I'heure actuelle, des investissements lourds dans de grandes plantations industrielles de cocotier pour la production d'huile de coprah ne semblent plus envisageables, du fait de la forte concurrence d'autres oléagineux, notamment le palmier. Le constat est loin d'être aussi négatif si l'on se place dans le cadre de plus petites structures de production pour lesquelles le coprah peut rester éventuellement la valorisation principale, à condition d'être associé à d'autres usages du cocotier. Celui-ci remplit alors de multiples fonctions.

* Espèce colonisatrice de milieux défavorables. Plante rustique, seule capable de se développer dans certaines conditions adverses, le cocotier est parfois la seule culture envisageable. Le cocotier est peu sensible au phénomène de "fatigue des sols ", ce qui traduit à la fois une susceptibilité aux parasites et maladies telluriques plus faible que la moyenne et une bonne aptitude à explorer le sol pour son approvisionnement en eau et en éléments minéraux.

* Rôle pivot dans les systèmes de production villageois. Désavantagé en situation de plantations industrielles du fait de sa moindre productivité agronomique, le cocotier conserve toutefois des atouts importants dans le cadre des plantations villageoises et paysannes, notamment par son rôle dans les systèmes de production des petits planteurs. La cocoteraie paysanne est souvent combinée à d'autres activités agricoles et assure tout à la fois un lissage des pics de travail et des besoins de trésorerie. Contrairement à d'autres graines oléagineuses, la noix de coco mûre peut supporter un délai d'attente de plusieurs semaines, voire de quelques mois, avant d'être exploitée, permettant à la main-d'œuvre familiale de faire face aux urgences des autres activités agricoles. Cela permet également de disposer de revenu monétaire mobilisable au moment où les dépenses se présentent.

* Contribution à la diversification de l'alimentation. Les noix servent prioritairement à la production de coprah, mais une partie d'entre elles est fréquemment consommée en vert. Elles sont alors autoconsommées, contribuant ainsi à la diversification de l'alimentation familiale, ou bien vendues à proximité, ce qui permet de générer un petit revenu additionnel immédiat.

* Rôle de marqueur foncier. Dans les sociétés traditionnelles africaines, le foncier est un bien collectif géré par les individus du groupe sous une forme usufruitière. Un droit d'exploitation est accordé aux agriculteurs, sous réserve qu'ils cultivent la terre. L'arrêt de l'exploitation de la terre entraîne la perte des droits accordés initialement, ce qui peut poser problème dans le cas des cultures annuelles. La situation est beaucoup plus simple avec les cultures pérennes : la plantation d'arbres confère au planteur un droit d'usage du sol pendant toute la durée d'exploitation du verger. L'aspiration générale à une sécurisation du foncier dans un contexte de gestion collective de la terre explique qu'il soit difficile pour un producteur d'éliminer les signes concrets d'un droit d'usage en abattant sa plantation âgée.

Des solutions innovantes doivent donc être imaginées pour assurer le renouvellement des vieux peuplements de cocotier : replantation avant abattage des vieux cocotiers, en culture pure ou dans le cadre d'associations de cultures ; remplacement par tranches du peuplement ancien ; replantation associée à la mise en place de cultures annuelles dans les interlignes pendant la période d'installation... 


\section{Replantation ou réhabilitation : quelle stratégie choisir ?}

L'amélioration ou la restauration de la compétitivité de la culture du cocotier dans une situation où la cocoteraie est jugée non rentable peut être obtenue de diverses façons, selon la nature du problème rencontré, le contexte socio-économique de production et la capacité du producteur à mettre en œuvre les solutions jugées les mieux adaptées. En clair, la stratégie à adopter sera d'autant plus appropriée qu'elle sera élaborée sur la base d'un bon diagnostic de la situation de départ et que l'itinéraire proposé reposera sur la mise en œuvre de moyens réellement mobilisables par le producteur.

\section{Démarche générale d'élaboration d'une stratégie adaptée}

Elle comporte plusieurs étapes qui, partant d'un diagnostic initial, conduisent à la définition d'un plan d'action adapté aux objectifs et aux moyens du producteur.

* Évaluation de la situation de départ : le diagnostic initial doit d'abord identifier la nature du problème rencontré. Si certaines situations sont extrêmement claires (par exemple, cas des ravages causés par une maladie mortelle ou par des cyclones), d'autres cas sont beaucoup moins faciles à analyser : c'est ainsi que certaines cocoteraies âgées sont déclarées séniles, à tort. Dans d'autres cas, le problème mis en avant par le producteur est d'ordre technique (rendement trop faible), alors qu'en fait des facteurs socio-économiques sont à l'origine du phénomène (le prix du coprah étant jugé trop faible par le planteur, celui-ci ne soigne plus sa cocoteraie, dont le rendement diminue). Quoi qu'il en soit, même mal exprimée, la demande reçue exprime la perception qu'a le producteur de son problème et renseigne bien sur la façon dont il envisage de le régler.

* Nécessité d'un diagnostic approprié : l'analyse doit porter sur la cocoteraie (aspects agronomiques et sanitaires), sur l'exploitation (capacité technique, moyens humains et financiers) et son environnement (contexte économique de production, fonctionnement de la filière). En résumé, il faut évaluer le potentiel de production de la cocoteraie et identifier la meilleure valorisation possible du produit dans les conditions actuelles.

* Solutions possibles : il existe un large choix de solutions techniques qui varient selon la nature du problème rencontré. La « meilleure solution " peut aller de l'abandon pur et simple de la culture du cocotier (en cas de perte définitive de compétitivité vis-à-vis d'autres productions ou en l'absence de matériel résistant/tolérant en zone soumise à une épidémie à maladie mortelle) à la replantation de la parcelle avec du matériel végétal performant et adapté (existence d'un bon potentiel agronomique et d'une demande soutenue de la part d'industries de transformation). Elle peut également consister à diversifier le système de culture (introduction de cultures associées) ou le système de production (intégration de l'élevage, ou démarrage d'une activité de transformation à petite échelle de la production).

* Gradation dans la mise en cuuvre des solutions choisies : elles peuvent être appliquées à différents niveaux d'intensité (doses de fertilisants par exemple) ou associer plusieurs techniques employées de façon combinée (fertilisation minérale et mulch organique ; replantation des parties de la cocoteraie les plus dégradées et réhabilitation des autres).

* Adéquation des solutions proposées à la réalité : les techniques préconisées doivent non seulement répondre au problème posé, mais également pouvoir être mises en œuvre par le 
producteur. Cela pose la question de leur accessibilité, en termes de disponibilité (intrants, technologies nouvelles) et de possibilités de les acquérir (épargne personnelle, accès au crédit), et celle du niveau technique des producteurs devant les appliquer (formation).

* Intérêt d'une vision prospective : la démarche présentée ci-dessus repose sur un diagnostic de la situation de départ et peut donc conduire à l'adoption de solutions conservatrices privilégiant le statu quo ante. Pour sortir de ce schéma statique, il est conseillé d'examiner les possibilités de développement ultérieur de l'exploitation et de la filière. Les perspectives de développement de l'activité " cocotier " au sein de l'exploitation sont à la base liées aux potentiels agronomique, humain, technique et financier de l'exploitation, mais elles dépendent aussi fortement de la stratégie définie par le producteur. En particulier, le choix entre réhabilitation, réhabilitation plus replantation ou replantation immédiate a une incidence importante sur le développement futur de l'exploitation : la réorientation de la production vers certains produits à forte valeur (noix à commercialiser en frais, noix à boire) dépend de la disponibilité de cocotiers jeunes et donc de la décision de réaliser de nouvelles plantations.

\section{Quand doit-on envisager de replanter?}

\section{* Pour répondre à une évolution de la demande}

La décision de replanter peut constituer la bonne réponse face à une modification de la demande, lorsque l'industrie de transformation située en aval de la filière évolue du coprah vers des produits de type fruitier (coco râpé, crème de coco...) issus du traitement en frais de noix entières. Ces nouveaux produits permettent de mieux valoriser la production que l'huile de coprah produite traditionnellement, mais leur développement implique de pouvoir assurer l'approvisionnement des unités de transformation en noix fraîches, ce qui pose à la fois un problème de qualité et une question de coût. Deux types de problèmes peuvent alors se poser : I'inadaptation du matériel végétal existant vis-à-vis de la préparation des nouveaux produits, qui conduit à son remplacement par du matériel présentant la qualité requise, et l'approvisionnement des unités de transformation en quantités suffisantes de noix arrivant à l'usine à un prix acceptable.

En effet, les coûts d'achat, de commercialisation et de transport sont fortement augmentés lorsque I'on passe du coprah, produit stable et allégé par un premier traitement post-récolte, aux noix fraîches, plus lourdes, volumineuses et fragiles. Les coûts de transport étant proportionnels au carré du rayon moyen de collecte, le transformateur a tout intérêt à s'approvisionner au plus près dans une zone présentant une bonne capacité de production. En général, l'unité s'est installée dans une zone de production existante réputée capable d'assurer son approvisionnement, mais la plupart du temps il sera intéressant d'améliorer la productivité de la cocoteraie prospectée afin de contracter le bassin de collecte. Cet effort d'intensification de la production passe par la réhabilitation des parcelles présentant le meilleur potentiel et par la replantation des autres ou encore la plantation des terres encore disponibles. La viabilité des projets de transformation en frais de la noix de coco dépendant donc, entre autres, de l'existence d'un potentiel de production suffisant, l'entreprise pourra avoir intérêt à s'impliquer dans des programmes de réhabilitation/replantation : fourniture d'intrants à crédit (engrais, plants), sur la base de contrats d'achat pluriannuels, par exemple. 


\section{* Pour assurer le maintien de la filière}

II existe malheureusement des situations où la production enregistre une chute marquée et irréversible, ce qui peut arriver lorsque les plantations atteignent un stade avancé de sénilité (voir définition ci-dessous), ou bien quand la cocoteraie est soumise à des épidémies sévères qui déciment des plantations entières (trois régions sont principalement concernées dans le monde par cette dernière situation, Mexique-Caraïbe, Togo-Ghana et Tanzanie-Mozambique).

Les cocoteraies séniles conjuguent tous les défauts pouvant être observés dans le cas des plantations âgées : forte mortalité cumulée induisant une faible densité, mauvais état général des palmiers, souffrant de graves carences et se défendant mal contre les agressions biotiques ou abiotiques, qui peut aller jusqu'à la misère physiologique qui précède la mort. II n'est pas rentable, ni même possible, de redresser de telles situations dans lesquelles les arbres très affaiblis ne peuvent soutenir la concurrence des adventices qui prolifèrent et deviennent incontrôlables.

La replantation des cocoteraies séniles n'est possible que si les conditions agro-climatiques sont encore suffisamment favorables à la culture du cocotier, ce qui exclut certaines zones devenues marginales (quelques régions d'Indonésie ou d'Afrique orientale) ou bien dont le sol est totalement épuisé par la culture précédente (dunes côtières du Bénin). À noter toutefois que, même dans ces conditions difficiles, la replantation peut être décidée pour atteindre des objectifs non directement productifs : fixation des sols fragiles et restauration de leur fertilité (cocotier associé à des légumineuses arbustives), ou maintien d'une cocoteraie côtière en zone touristique...

La replantation (ou nouvelle plantation) en zone soumise à une épidémie ou sinistrée par un cataclysme nécessite de pouvoir disposer de matériel végétal résistant ou tolérant à la maladie ou au facteur abiotique, quand ce matériel existe (par exemple, utilisation d'hybrides de Grand Vanuatu dans la zone du Ghana affectée par le jaunissement mortel, ou encore choix d'écotypes résistants aux cyclones en zone côtière d'Afrique austro-orientale). À noter que la notion de résistance est souvent très relative et que, la plupart du temps, elle est assortie d'une évaluation du risque encouru : c'est ainsi que, au Ghana, l'espérance de vie des nouvelles plantations situées en zone de jaunissement mortel est estimée à 15 à 20 ans environ au minimum, dans l'état actuel de nos connaissances.

* Bien que rarement exploitées, il existe quelques possibilités de tirer un petit revenu de l'opération d'abattage précédant la replantation. Le bourgeon terminal du cocotier constitue un met recherché (cœur de palmier ou chou coco) dont la vente rapporte un revenu de l'ordre de trois à dix fois le coût d'un plant de cocotier amélioré prêt à replanter. Le stipe des vieux cocotiers donne un " bois " dense et coloré qui peut être parfaitement valorisé en menuiserie et en ébénisterie. Malheureusement, l'exploitation de ce " bois " nécessite des outils et des techniques très particulières, ce qui explique que son usage soit si peu répandu. L'on retiendra tout de même l'idée qu'il serait intéressant de prévoir un volet de valorisation des cocotiers abattus dans tout programme important de replantation (projet industriel ou programme villageois organisé à l'échelle d'une région de production). 
La replantation ne constitue pas toujours la meilleure réponse à une baisse importante de la production d'une cocoteraie âgée : il suffit parfois d'une simple réhabilitation pour améliorer sensiblement sa productivité [4]. L'intensification de l'itinéraire de production permet d'obtenir un gain de production, certes moindre, mais rapide (il n'est pas nécessaire d'abattre les arbres) et ne demande qu'un investissement monétaire limité (tableau 4).

\section{* Quand réhabiliter?}

En pratique, l'opération est envisageable quand les cocoteraies sont peu productives, sans être séniles, et qu'elles présentent encore un potentiel de production acceptable. Le choix de cette solution demande d'avoir réalisé un diagnostic agronomique rigoureux : évaluation précise du peuplement, de l'état sanitaire des arbres, de leur niveau de production actuel, de la situation de la nutrition minérale ; comparaison de la production observée avec le potentiel connu pour ce matériel végétal dans ce type de situation. Un bas niveau de rendement n'est pas rédhibitoire si les arbres n'ont pas souffert de carences débilitantes et si l'analyse du contexte de production permet d'interpréter la faiblesse des rendements. L'évaluation est avant tout pragmatique, mais elle peut être affinée ou validée par des analyses complémentaires de type « diagnostic foliaire ».

\section{* En quoi consiste-t-elle?}

La réhabilitation doit assurer l'accroissement de la production en levant, totalement ou partiellement, les contraintes pesant sur elle : élimination des adventices ou du recrû forestier qui sont en compétition pour l'alimentation édaphique, suppression des carences les plus importantes par une fertilisation minérale adaptée, voire, sur un plus long terme, restauration de la fertilité (engrais minéraux et organiques, restitution des déchets de production).

\section{* Comment agit-elle?}

L'opération a pour premier résultat une amélioration sensible de la végétation puis, avec un certain décalage dépendant à la fois de la situation de départ et des moyens appliqués, la production de coprah s'accroît sur les plans quantitatif (nombre de noix par arbre) et qualitatif (ratio coprah par noix). Sur la base des résultats obtenus en réhabilitation aux Philippines sur de vastes surfaces (300 $000 \mathrm{ha})$, l'application de fertilisants pendant 3 ans a permis de doubler les rendements à l'hectare au bout de 2 ans, la production restant à ce niveau pendant 3 ans et ne revenant à la valeur initiale que lentement après l'arrêt total des apports fertilisants.

\section{Autres solutions envisageables}

\section{* Les associations de culture à base de cocotier}

Il est possible d'associer d'autres cultures au cocotier, au démarrage des plantations ou à l'âge adulte. À la plantation, l'installation de cultures vivrières dans les interlignes est possible car le cocotier, encore peu développé, occupe un espace réduit. Cette solution permet de valoriser la terre à ce stade non productif et constitue une solution efficace pour assurer le contrôle des adventices qui disposent d'excellentes conditions pour se développer et concurrencer les jeunes palmiers. À l'âge adulte, le cocotier occupe tout l'espace disponible et empêche le développement de la plupart des espèces concurrentes par interception de la plus grande part des radiations 
incidentes. Cependant, à partir de 10-15 ans, avec l'élévation des couronnes des palmiers, la transmission de la lumière sous cocotier s'améliore et certaines cultures intercalaires deviennent possibles : cacaoyer, certains fruitiers, quelques racines, légumineuses arbustives. Ces dernières permettent d'assurer une production de bois (perches, bois de feu) tout en assurant un apport d'azote organique bénéfique au développement du cocotier.

\section{* La cocoteraie adulte est tout à fait compatible avec d'autres productions}

Ce sont l'élevage sous cocotier, qui est bénéfique à la plante (entretien des adventices assuré, restitution de déjections constituant un engrais organique intéressant), le maraîchage aux abords des grandes villes (sur planches de compost végétal installées sous de vieux cocotiers assurant l'ombrage) ou encore le développement d'activités de transformation des produits du cocotier. L'intégration de la transformation dans l'exploitation agricole permet une meilleure valorisation de la production (valeur ajoutée accrue) tout en sécurisant le revenu du producteur par la diversification des activités : extraction d'huile artisanale, fabrication de savon par le procédé à froid, tressage d'objets de vannerie, etc.

* Rappelons enfin qu'il existe des cas où le planteur n'a aucun intérêt à maintenir sa cocoteraie insuffisamment productive et encore moins à investir pour améliorer sa productivité. Dans de telles situations, le producteur doit s'engager dans un processus de modification de son système de culture, qui passe par l'élimination du cocotier. Le passage au nouveau système sans cocotier peut être progressif, ce qui permet à l'exploitant de conserver un certain revenu pendant la période de changement et lui laisse le temps de s'adapter aux nouveaux itinéraires techniques.

\section{Synthèse méthodologique}

L'amélioration de la rentabilité de la cocoteraie peut prendre plusieurs voies, selon la nature du problème rencontré, le contexte agro-socio-économique de production, les objectifs du producteur et sa capacité à mettre en œuvre les solutions apparaissant les mieux adaptées. La diversité des situations rencontrées est telle qu'il est impossible de dresser une liste exhaustive de solutions standard pouvant répondre à telle ou telle question posée. En revanche, une démarche a été proposée qui doit permettre au décideur (petit ou gros planteur, responsable du développement rural) d'élaborer une stratégie adaptée de réhabilitation.

Quelques situations types et les solutions correspondantes pouvant être proposées sont décrites à titre d'exemple dans le tableau 5.

\section{CONCLUSION}

Du fait du taux de renouvellement de plus en plus faible des plantations et replantations depuis plusieurs décennies, la cocoteraie mondiale est vieillissante et sa productivité décline progressivement, au moment où celle d'autres plantes oléagineuses a très fortement augmenté. L'ère des grands programmes de plantation de cocotiers destinés à la production d'huile de coprah semble définitivement révolue. Les cocoteraies ne sont pas condamnées pour autant. Elles continuent d'assurer de multiples fonctions dans un contexte villageois et dans des zones marginales où d'autres activités productives sont difficilement envisageables, grâce à la diversité des utilisations de la noix. L'intérêt des cocoteraies villageoises réside notamment dans la demande en produits issus de la transformation en frais et dans la consommation des noix vertes, mais la 
production de coprah y garde malgré tout une place de choix du fait de l'existence de circuits commerciaux établis et de la souplesse offerte dans le déroulement de l'activité en termes de calendrier de travaux. Dans ces conditions, des programmes ciblés de réhabilitation de cocoteraies partiellement dégradées et de plantation ou replantation de cocoteraies séniles ou fortement dégradées peuvent avoir un impact très positif sur le bien-être des populations concernées. II convient alors d'être très sélectif dans le choix des techniques de réhabilitation et de replantation pour que celles-ci répondent au mieux aux besoins identifiés localement.

\section{REFERENCES}

1. Oil World. Annuaire statistique.

2. TOMICH T, MAWARDI M (1995). Evolution of palm oil trade policy in Indonesia. Elaeis, 1 : 87-102.

3. VOITURIEZ T, DE NUCÉ M (1997). L'avenir contrasté du marché des huiles lauriques. Plantations, Recherche, Développement, $6: 378-84$.

4. SIMON D (1998). Coconut sector development project ; evaluation report AFD : 65.

Illustrations

\begin{tabular}{|lrrrrr}
\hline & 1961 & 1971 & 1981 & 1991 & 1999 \\
\hline Afrique & 430 & 502 & 594 & 643 & 664 \\
Côte d'lvoire & 4 & 11 & 37 & 50 & 30 \\
Ghana & 29 & 36 & 52 & 27 & 54 \\
Madagascar & 8 & 12 & 26 & 32 & 33 \\
Mozambique & 105 & 105 & 105 & 105 & 105 \\
Nigeria & 28 & 34 & 35 & 37 & 35 \\
Tanzanie & 170 & 220 & 260 & 304 & 310 \\
Autres & 86 & 84 & 79 & 88 & 94 \\
Asie & 3980 & 5.520 & 7400 & 8260 & 8720 \\
Indonésie & 1130 & 1320 & 1890 & 2270 & 2550 \\
Philippines & 1200 & 2050 & 3220 & 3100 & 3050 \\
Océanie & 503 & 491 & 571 & 571 & 527 \\
Monde & 5230 & 6990 & 8910 & 10090 & 10490
\end{tabular}

Tableau 1. Évolution des superficies cultivées en cocotier en Afrique entre 1961 et 1999 (en milliers d'hectares)Source : FAOSTAT. 


\begin{tabular}{|lccccc}
\hline & 1961 & 1971 & 1981 & 1991 & 1999 \\
\hline Production d'huile de copra h & 1,86 & 2,37 & 2,80 & 2,96 & 2,78 \\
Production d'huile de palmiste & 0,49 & 0,52 & 0,75 & 1,59 & 2,53 \\
Production toutes huiles & 24,6 & 37,1 & 54,5 & 78,2 & 102,2 \\
Part de Phuile de coprah (\%) & 7,6 & 6,4 & 5,1 & 3,8 & 2,7
\end{tabular}

Tableau 2. Évolution des parts de l'huile de coprah dans la production totale d'huiles végétales (en millions de tonnes)Source : FAOSTAT.

\begin{tabular}{|lrrrrr}
\hline & 1961 & 1971 & 1981 & 1991 & 1999 \\
\hline Cocoteraies & 356 & 339 & 314 & 293 & 265 \\
Palmeraies & 409 & 652 & 1296 & 1863 & 2260 \\
\hline
\end{tabular}

Tableau 3. Évolution des rendements en huile des cocoteraies et palmeraies entre 1961 et 1999 (en $\mathrm{kg} / \mathrm{ha}$ )

Source : FAOSTAT.

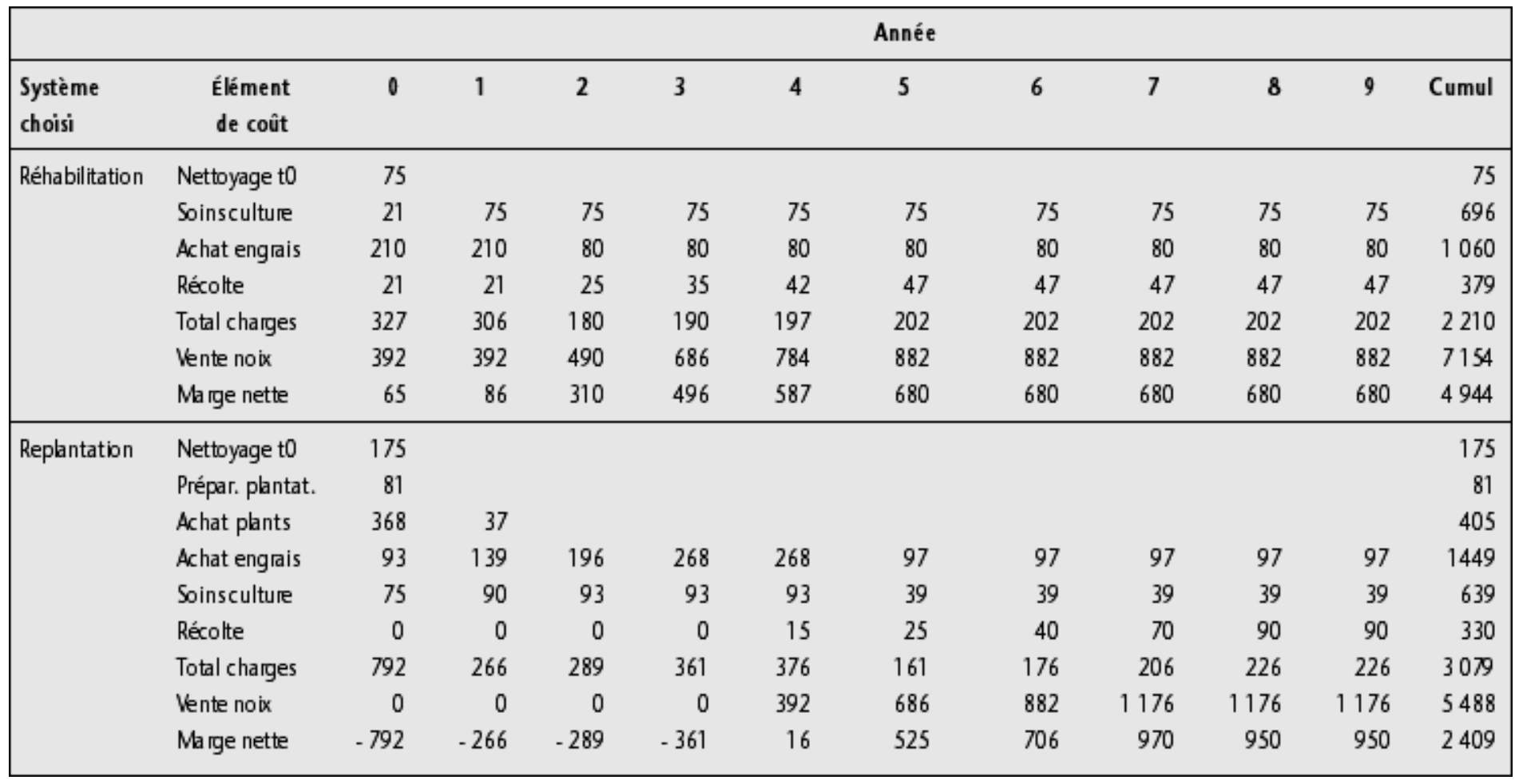

Tableau 4. Rentabilité comparée des systèmes de réhabilitation et de replantation. (Cas du Ghana, Western Region, 1998 ; coûts par hectare, exprimés en milliers de cedis) 



\begin{tabular}{|c|c|c|c|}
\hline Nature du problème & $\begin{array}{l}\text { Contexte ag ro-socio-économique } \\
\text { de production }\end{array}$ & $\begin{array}{l}\text { Solution pouvant } \\
\text { être proposée }\end{array}$ & $\begin{array}{l}\text { Atouts }(+) \text { et contrain tes }(-) \\
\text { tech niques et économiques }\end{array}$ \\
\hline \multirow[t]{5}{*}{$\begin{array}{l}\text { Cocotiers peu productifs } \\
\text { mais présentant encore un potentiel }\end{array}$} & $\begin{array}{l}\text { Zone adaptée à la culture } \\
\text { et disposant de débouchés } \\
\text { pour les produits }\end{array}$ & Intensification-réhabilitation & $\begin{array}{l}\text { (+) Coût limité, retour rapide; } \\
\text { système de culture inchangé }\end{array}$ \\
\hline & $\begin{array}{l}\text { Copra h mal valorisé } \\
\text { mais il n'existe pas d'altemative }\end{array}$ & Intensification-réhabilitation & $\begin{array}{l}\text { (-) Progrès limité ; } \\
\text { matériel végétal inchangé }\end{array}$ \\
\hline & Il existe d'a utres filières & Cultures associées sous cocotier & (+) Diversification, \\
\hline & de commercialisation concernant & Productions associées (bois, élevage) & sécurisation du revenu \\
\hline & d'autres cultures de rente & Abattage et changement de culture & $\begin{array}{l}\text { (-) Investisssement requis, } \\
\text { avec retour tardif; ; apprentissage } \\
\text { de nouveaux savoir-faire }\end{array}$ \\
\hline \multirow[t]{3}{*}{ Copra h peu rémunérateur } & $\begin{array}{l}\text { Existence d'une demande } \\
\text { pour d'autres produits } \\
\text { issus du cocotier }\end{array}$ & $\begin{array}{l}\text { Diversification des marchés } \\
\text { (avec ou sans intensification, } \\
\text { réhabilitation) }\end{array}$ & $\begin{array}{l}\text { (+) Amélioration, diversification } \\
\text { et sécurisation du revenu } \\
\text { (-) Progrès limité (sauf si } \\
\text { ensemble des mesures) }\end{array}$ \\
\hline & $\begin{array}{l}\text { Existence d'une demande } \\
\text { pourd'autres productions }\end{array}$ & $\begin{array}{l}\text { Intégration de b transformation } \\
\text { (transformation artisanale) }\end{array}$ & $\begin{array}{l}\text { (+) Amélioration-diversification } \\
\text { et sécurisation du revenu }\end{array}$ \\
\hline & $\begin{array}{l}\text { pouvant être obtenues en association } \\
\text { avec le cocotier }\end{array}$ & $\begin{array}{l}\text { Cultures associées sous cocotier } \\
\text { Productions associées (bois, élevage) }\end{array}$ & $\begin{array}{l}\text { (-) Investissement fina ncier } \\
\text { et humain (apprentissage } \\
\text { des nouveaux métiers) }\end{array}$ \\
\hline \multirow[t]{2}{*}{ Nouvelle demande } & $\begin{array}{l}\text { La production actuele n'est pas } \\
\text { adaptée à la nouvelle demande }\end{array}$ & $\begin{array}{l}\text { Réhabilitation-intensification- } \\
\text { replantation graduelle des pa relles } \\
\text { ks plus âgées (amélioration } \\
\text { des rendements, de la production } \\
\text { et du coprah par noix) }\end{array}$ & $\begin{array}{l}\text { (+) Coût modéré, effet rapide; } \\
\text { investissement graduel (replantation) } \\
\text { (-) La production ne satisfait pas } \\
\text { totalement la demande }\end{array}$ \\
\hline & $\begin{array}{l}\text { La cocoteraie ex istante ne peut foumir } \\
\text { a demande en quantité et en qualité }\end{array}$ & $\begin{array}{l}\text { Replantation avec une variété } \\
\text { prod uctive et adaptée }\end{array}$ & $\begin{array}{l}\text { (+) La production répondra } \\
\text { exactement à la demande } \\
\text { (-) Coût élevé, satisfaction différée } \\
\text { de la demande, retour lointain }\end{array}$ \\
\hline Cocoteraie sénile & $\begin{array}{l}\text { Zone adaptée à la culture } \\
\text { Solsépuisés } \\
\text { Zone devenue impropre à la culture }\end{array}$ & $\begin{array}{l}\text { Replantation } \\
\text { Replantation en association } \\
\text { avec légumineuses arbustives } \\
\text { (restauration de la fertilité) }\end{array}$ & $\begin{array}{l}\text { (+) Restauration d'un fort potentiel } \\
\text { de production } \\
\text { (-) Coût élevé, retour bintain }\end{array}$ \\
\hline Cocoteraie ravagée par une maladie & $\begin{array}{l}\text { Zone adaptée à la culture } \\
\text { Culture essentielle pour la zone } \\
\text { Pasde culture substituable } \\
\text { Possibilité de cultures alternatives }\end{array}$ & $\begin{array}{l}\text { Replantation avec du matériel } \\
\text { toléra nt-résistant } \\
\text { Passage à d'autres cultures/ } \\
\text { systèmes de prod uction }\end{array}$ & $\begin{array}{l}\text { (+) Restauration du potentiel } \\
\text { de production } \\
\text { (-) Coût élevé, retour bintain } \\
\text { (+) Restauration d'un potentiel } \\
\text { de production avec diversification } \\
\text { (-) Coût élevé, retour bintain }\end{array}$ \\
\hline $\begin{array}{l}\text { Cocoteraie ravagée } \\
\text { par catastrophes naturelles (cyclones) }\end{array}$ & $\begin{array}{l}\text { Zone adaptée à la culture } \\
\text { Culture essentielle pour la zone } \\
\text { Pasde culture substituable } \\
\text { Possibilités de substitution }\end{array}$ & $\begin{array}{l}\text { Replantation avec du matériel } \\
\text { toléra nt-résistant } \\
\text { Pa ssage partiel ou intégral } \\
\text { à d'autres cultures/ } \\
\text { systèmes de prod uction }\end{array}$ & $\begin{array}{l}\text { (+) Restauration du potentiel } \\
\text { de production } \\
\text { (-) Coût élevé, retour bintain } \\
\text { (+) Restauration d'un potentiel } \\
\text { de production diversifié ou modifié } \\
\text { (-) Coût élevé, pour un retour } \\
\text { plus ou moins bintain sebon solution }\end{array}$ \\
\hline
\end{tabular}

Tableau 5. Analyse de quelques solutions techniques pouvant être proposées dans des situations problématiques types 